\section{Prismatic correction of residual esotropia of 20 prism dioptres or less after full hypermetropic correction}

\begin{abstract}
Purpose To assess the efficacy of prismatic correction of residual esotropia $\leqslant 20$ prism dioptres (PD) after full hypermetropic correction in patients with partially accommodative esotropia.

Methods Medical records of 64 patients who received prismatic correction for residual esotropia $\leqslant 20$ PD were reviewed. Outcomes were considered successful if patients maintained orthotropia or esophoria for at

least 1 year and did not require surgery.

Factors including age, sex, visual acuity, refractive errors, amount of deviation, sensory status, and the presence of amblyopia were analysed and compared between the success and failure groups.
\end{abstract}

${ }^{1}$ Department of Ophthalmology, Seoul National University College of Medicine, Seoul, Korea

${ }^{2}$ Department of Ophthalmology, Seoul National University Bundang Hospital, Seongnam, Korea

Correspondence: J-M Hwang, Department of Ophthalmology, Seoul National University Bundang Hospital, Seoul National University College of Medicine, 300 Gumi-dong, Bundang-gu, Seongnam, Gyeonggi 463-707, Korea

Tel: + 82317877372 ;

Fax: + 82317874057

E-mail: hjm@

snu.ac.kr

Received: 7 August 2008 Accepted: 16 December 2008

Published online: 30 January 2009
Results Prismatic correction was successful in 28 of 64 patients (44\%). The success group showed better results both with Worth 4-dot test $(P=0.001$ at distance and $P=0.046$ at near) and Randot stereo test $(P=0.003$ for dots and $P=\mathbf{0 . 0 0 0}$ for animals). Success rate increased to $58 \%$ without amblyopia, 72 and $93 \%$ with normal fusional response at near and at distance with Worth 4 dot test respectively, and $92 \%$ with stereoacuity of $800 \mathrm{~s}$ of arc or better. In all patients in success group, fusion and stereoacuity improved or maintained during follow-up.

Conclusions Prismatic correction was successful in $44 \%$ of the patients with residual esotropia $\leqslant 20$ PD and the success group showed an improved or stable sensory status with time. With a baseline fusion on Worth 4-dot test or stereopsis of $800 \mathrm{~s}$ of arc or better, prismatic correction could be considered as the first-line treatment.
SB Han ${ }^{1}$ and J-M Hwang ${ }^{1,2}$

Eye (2009) 23, 2052-2055; doi:10.1038/eye.2008.424; published online 30 January 2009

Keywords: amblyopia; binocularity; fusion; partially accommodative esotropia; prismatic correction; stereopsis

\section{Introduction}

The first step in the management of esotropia associated with hyperopia is the prescription of glasses to fully correct the hyperopia. ${ }^{1,2}$ If the esotropia is significantly undercorrected with glasses, it is termed as partially accommodative esotropia. ${ }^{2}$ The conventional treatment for partially accommodative esotropia is surgical correction for the residual deviation. ${ }^{1-4}$ However, it is unclear that all residual esotropia should be surgically corrected. To the best of our knowledge, no previous report has shown whether a small angle of residual esotropia should be surgically treated or could be mananged only with a non-surgical treatment such as prism glasses. The purpose of this study is to assess the efficacy of prismatic correction instead of surgery for partially accommodative esotropia $\leqslant 20$ prism dioptres (PD) after a full hyperopic correction.

\section{Materials and methods}

Sixty-four (28 male, 36 female) consecutive children $\leqslant 15$ years of age who underwent a prismatic correction for partially accommodative esotropia $\leqslant 20$ PD after a full hypermetropic correction between June 2004 and March 2007 were retrospectively studied. Patients were excluded if they had residual 
esotropia > 20 PD after a full hypermetropic correction, hypermetropia $<+1.50$ dioptres, history of prior strabismus surgery, dissociated vertical deviation, oblique muscle dysfunction, any paralytic or restrictive esotropia, ocular pathology, chromosomal anomaly, systemic disorder such as a congenital anomaly, neurological disorder, developmental abnormality, or with a follow-up shorter than 1 year after a prismatic correction.

The medical records were retrospectively reviewed for age, sex, best-corrected visual acuity (BCVA), refractive errors with cycloplegic refraction, amount of esotropia measured by alternate prism cover test both at distance and near with corrections, Randot stereotest, and Worth 4-dot test at 6 and $0.33 \mathrm{~m}$ before and after prismatic correction (Table 1).

All patients underwent a complete ophthalmologic examination, including visual acuity test and cycloplegic refraction with cyclopentolate hydrochloride $1 \%$, followed by a prescription of glasses to fully correct the hypermetropia. Prism and alternate cover test with accommodative targets for fixation at 0.33 and $6 \mathrm{~m}$ were conducted in most of the patients, and a modified Krimsky method at $0.33 \mathrm{~m}$ was conducted in a few uncooperative patients, by one of the authors (JMH). Prism was prescribed and ground into the lens according to residual esotropia. Patients with a difference of two lines or more of visual acuity in each eye were considered to have amblyopia and underwent part-time or full-time occlusion along with spectacle wearing. Sensory status was evaluated using the Randot stereotest and the Worth 4-Dot test at distance and near. Visual acuity test, Randot stereotest, and Worth 4-dot test were performed when possible. In Worth-4-dot test, only a fusional response of four light visible was considered normal. Follow-up examinations were

Table 1 Demographic characteristics, BCVA, refractive errors, and angle of deviation of the patients in the success and failure groups

\begin{tabular}{lccc}
\hline & Success group & Failure group & P-value \\
\hline Age (months) & $65.3 \pm 31.1$ & $50.6 \pm 37.2$ & $0.090^{\mathrm{a}}$ \\
Sex (M: F) & $14: 14$ & $14: 22$ & $0.263^{\mathrm{b}}$ \\
BCVA $^{\mathrm{c}}$ (right) & $0.34 \pm 0.14$ & $0.34 \pm 0.14$ & $0.233^{\mathrm{a}}$ \\
BCVA $^{\mathrm{c}}$ (left) & $0.34 \pm 0.22$ & $0.36 \pm 0.29$ & $0.830^{\mathrm{a}}$ \\
SEQ (right, dioptres) & $+4.1 \pm 1.3$ & $+4.2 \pm 2.2$ & $0.783^{\mathrm{a}}$ \\
SEQ (left, dioptres) & $+3.6 \pm 1.2$ & $+4.0 \pm 2.1$ & $0.308^{\mathrm{a}}$ \\
Dcc & $9.6 \pm 6.8$ & $13.1 \pm 3.1$ & $0.085^{\mathrm{a}}$ \\
Ncc & $11.2 \pm 5.4$ & $14.0 \pm 4.4$ & $0.098^{\mathrm{a}}$ \\
\hline
\end{tabular}

$\mathrm{BCVA}=$ best-corrected visual acuity; Dcc = distant deviation with correction; $\mathrm{F}=$ female; $\mathrm{Ncc}=$ near deviation with correction; $\mathrm{M}=$ male; $\mathrm{SEQ}=$ spherical equivalent.

aStudent's $t$-test.

${ }^{\mathrm{b}} \chi^{2}$-test.

'BCVA in LogMAR. performed at $1,3,6$, and 12 months after prescription of prism glasses.

If orthotropia or esophoria was maintained with prism glasses for $\geqslant 1$ year without surgery and showed no deterioration of visual function, the cases were assigned to the success group. Otherwise, they were assigned to the failure group.

The clinical features of the two groups were compared using the Fisher's exact test or $\chi^{2}$-test to examine the effect of sex, results of Worth 4-dot test, and presence of amblyopia at the beginning of prism correction on the success rate. Student's $t$-test (independent and paired samples' $t$-tests) was used to determine the effect of age, BCVA, esodeviation, and stereoacuity at the beginning of prism correction. SPSS software for Windows (V13.0; SPSS Inc., Chicago, IL, USA) was used in every statistical analysis. A $P$-value of less than 0.05 was considered statistically significant.

\section{Results}

The mean age at the beginning of prismatic correction was $57.0 \pm 35.2$ months (mean $\pm \mathrm{SD}$, range: $4-210$ months). Twenty-eight children (44\%) maintained orthotropia or esophoria with prism glasses and were classified in the success group. In the success group, the mean follow-up was 20.6 \pm 5.7 months (range: $12-33$ months). Age at the beginning of prismatic correction was $65.3 \pm 31.1$ months in the success group and $50.6 \pm 37.2$ months in the failure group $(P=0.090)$. In patients of success group, no or minimal (2-6 PD) increase in the prismatic correction was necessary during follow-ups, whereas those in failure group needs substantial increase of esotropia $\geqslant 10$ PD, and therefore had surgery.

Success rate was not different according to the sex (50\% in men vs $39 \%$ in women), BCVA, or difference in hypermetropia between the two groups. Mean residual deviation after hypermetropic correction at distance was $9.6 \pm 6.8 \mathrm{PD}$ in success group and $13.1 \pm 3.1 \mathrm{PD}$ in failure group, and those at near were 11.2 \pm 5.4 PD in success group and 14.0 $\pm 4.4 \mathrm{PD}$ in failure group (Table 1 ; $P>0.05)$. However, when the patients were divided by residual esotropia $\leqslant 10 \mathrm{PD}$ and $>10 \mathrm{PD}$, the success rate was significantly higher in the group $\leqslant 10 \mathrm{PD}$, both at distance and near (Table 2 ). In the group $\leqslant 10$ PD, success rate was $62 \%$ (8 of 13 patients) at distance and $67 \%$ (8 of 12 patients) at near, in contrast to $22 \%$ (5 of 23 patients) at distance and $22 \%$ (6 of 27 patients) in the group > 10 PD.

Forty-eight percent (10 of 21 patients in whom visual acuity could be measured) of patients in the failure group vs $17 \%$ ( 3 of 18 patients) in the success group had amblyopia before prism correction, with a statistically 
Table 2 The ratio of patients with residual esotropia $\leqslant 10$ PD $v s>10$ PD in success and failure groups

\begin{tabular}{lcccc}
\hline & Success group $(\mathrm{n})$ & Failure group $(\mathrm{n})$ & Odds ratio $(95 \%$ CI $)$ & P-value \\
\hline Dcc ( $\leqslant 10$ PD: $>10$ PD) & $8: 5$ & $5: 18$ & $5.76(1.29-25.6)$ & $0.022^{\mathrm{a}}$ \\
Ncc ( $\leqslant 10$ PD: $>10$ PD) & $8: 6$ & $4: 21$ & $7.00(1.56-31.5)$ & $0.011^{\mathrm{a}}$ \\
\hline
\end{tabular}

$\mathrm{CI}=$ confidence interval; $\mathrm{Dcc}=$ distant deviation with correction; $\mathrm{Ncc}=$ near deviation with correction; $\mathrm{PD}=$ prism dioptre.

Patients with residual esotropia > 10 PD had significantly higher risk of failure of prismatic correction, both at near and distance.

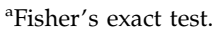

Table 3 Presence of amblyopia and sensory status as prognostic factors of prismatic correction

\begin{tabular}{lcccc}
\hline & Success group & Failure group & Odds ratio (95\% CI) & P-value \\
\hline Amblyopia $^{\mathrm{a}}$ (normal: amblyopia) & $15: 3$ & $10: 11$ & $4.55(1.01-20.4)$ & $0.043^{\mathrm{b}}$ \\
W4D at near $^{\mathrm{a}}$ (normal: abnormal) & $13: 7$ & $5: 11$ & $4.09(1.01-16.6)$ & $0.046^{\mathrm{b}}$ \\
W4D at distance $^{\mathrm{b}}$ (normal: abnormal) & $12: 8$ & $1: 15$ & $22.5(2.46-206)$ & $0.001^{\mathrm{b}}$ \\
Randot test (number of dots) $^{\mathrm{c}}$ & $1.80 \pm 1.91$ & $0.21 \pm 0.80$ & $0.003^{\mathrm{d}}$ \\
Randot test (number of animals) $^{\mathrm{c}}$ & $1.40 \pm 1.23$ & $0.14 \pm 0.54$ & & $0.000^{\mathrm{d}}$ \\
\hline
\end{tabular}

$\mathrm{CI}=$ confidence interval; W4D = Worth 4-dot.

The success rate increased with better baseline sensory status and decreased with the presence of baseline amblyopia.

an number of patients.

bFisher's exact test.

'In number of correct answer.

'Student's $t$-test (independent samples' $t$-test).

significant difference $(P=0.043)$. Success rate increased to $58 \%$ (15 of 26 patients) in children without amblyopia.

Success rate was significantly higher with $72 \%$ (13 of 18) in patients who showed normal fusional response at near, 93\% (13 of 14) at distance, with Worth 4-dot test after hypermetropic correction. Randot stereotest revealed that children in success group had significantly better stereopsis at the beginning of the prismatic correction. In success group, 60\% (12 of 20) of patients in whom Randot stereotest was possible showed stereopsis of $800 \mathrm{~s}$ of arc or better before prismatic correction. In contrast, only 1 of $14(7 \%)$ patients in failure group had stereopsis of $800 \mathrm{~s}$ of arc, and the rest showed no stereopsis with Randot stereotest. Twelve of thirteen $(92 \%)$ patients with stereopsis of $800 \mathrm{~s}$ of arc or better showed favourable response to prismatic correction (Table 3).

In all of the patients of success group, fusion and stereoacuity improved or maintained during follow-up. One year after prismatic correction, 33\% (6 of 18 patients) revealed improvement of Worth 4-dot test and 39\% (7 of 18 patients) showed an improvement in stereoacuity, and 14 of $22(64 \%)$ patients in whom stereotest was measurable had stereoacuity of $800 \mathrm{~s}$ or better. The rest showed the same sensory status results as those obtained 1 year before. Success group subjects recognized mean $1.80 \pm 1.91$ dots and $1.40 \pm 1.23$ animals at the initial examination, and $2.72 \pm 2.49$ dots and $1.83 \pm 1.42$ animals after 1 year (Table 4).
Table 4 The result of Worth 4-dot test and Randot stereo test at the beginning and 1 year after the prismatic correction in success group

\begin{tabular}{lccc}
\hline & At the beginning & 1 year after & P-value \\
\hline Randot test $(\text { dot })^{\mathrm{b}}$ & $1.80 \pm 1.91$ & $2.72 \pm 2.49$ & $0.010^{\mathrm{a}}$ \\
Randot test $(\mathrm{animal})^{\mathrm{b}}$ & $1.40 \pm 1.23$ & $1.83 \pm 1.42$ & $0.007^{\mathrm{a}}$ \\
\hline
\end{tabular}

aPaired samples' $t$-test.

${ }^{\mathrm{b}}$ In number of patients.

\section{Discussion}

As a non-surgical treatment for partially accommodative esotropia, miotics have been reported to be helpful in a small amount of residual esotropia. ${ }^{5}$ However, side effects, such as development of iris cyst or transient visual blurring, were frequent. ${ }^{2}$ Moreover, gradual increase in esotropia was still observed in spite of miotic therapy. ${ }^{6}$

In this study, we investigated the efficacy of another non-surgical treatment for partially accommodative esotropia: prismatic correction. Prismatic correction was successful in $44 \%$ patients with residual esotropia $\leqslant 20$ $\mathrm{PD}$, and 62 (at distance) and 67\% (at near) in patients with residual esotropia $\leqslant 10 \mathrm{PD}$. Success rate increased to $58 \%$ in patients without amblyopia, $72 \%$ with normal fusional response at near, and 93\% with normal fusional response at distance. Success rate was highest at $92 \%$ with stereopsis of $800 \mathrm{~s}$ of arc or better (Table 3). These 
results correspond with the earlier reports that nil stereopsis posed 17 times greater risk of surgery in accommodative esotropia, ${ }^{7}$ or that the presence of amblyopia had positive correlation with incomplete spectacle control or deterioration of accommodative esotropia. ${ }^{8,9}$ Birch et $a l^{10}$ also reported that subnormal stereopsis had positive correlation with the risk of developing accommodative esotropia in hypermetropic children. Swan ${ }^{11}$ reported that poor binocular function significantly increased the risk of requiring surgery in accommodative esotropia. In summary, we can predict the efficacy of prismatic correction based on the sensory status when we start prismatic correction, as patients with better baseline sensory status showed lower change of compensation of prismatic correction. The prismatic correction can be strongly recommended in a child with better sensory status.

Regarding the change of sensory status with the prismatic treatment, fusional responses and stereopsis improved in $\geqslant 1 / 3$ of the patients after 1 year of treatment and was maintained in the remaining patients. We found that $64 \%$ of patients in success group showed stereoacuity of $800 \mathrm{~s}$ or better 1 year after prismatic correction. Kushner ${ }^{12}$ showed that 189 of 341 (55\%) patients with partially accommodative esotropia had stereopsis of $800 \mathrm{~s}$ or better 5 years after surgery, and the rate decreased to $36 \%$ (8 of 22) in patients who were surgically overcorrected. In cases where patients underwent surgery for partially accommodative esotropia, Hwang et $a l^{1}$ reported that the portion of patients who showed positive fusional response increased by 14-48\% 1 year after surgery. Fawcett and Birch $^{13}$ showed that $3 / 4$ of patients with accommodative esotropia showed abnormal binocular response even after achieving successful eye alignment with or without surgery. Therefore, the effect of prismatic correction on sensory status is beneficial and not worse compared to surgery.

This study showed the value of non-surgical treatment of non-accommodative component of partially accommodative esotropia $\leqslant 20 \mathrm{PD}$ to improve or maintain sensory status. To the best of our knowledge, there has been no study, which investigated the efficacy of prismatic correction of residual esodeviation after hypermetropic correction. This is the first study to show the efficacy of prismatic correction as a non-surgical treatment in partially accommodative esotropia, and definitively show when prismatic correction can be successful. The results of this study suggest that in cases with baseline fusion on Worth 4-dot test or stereopsis of $800 \mathrm{~s}$ of arc or better, prismatic correction should be tried before a surgical cocrrection.

In conclusion, prismatic correction in a partially accommodative esotropia was successful in $44 \%$ of the patients, who showed improved or maintained sensory status with the prismatic correction. The success rate increased with better baseline sensory status and decreased with the presence of baseline amblyopia.

\section{References}

1 Hwang JM, Min BM, Park SC, Oh SY, Sung NK. A randomized comparison of prism adaptation and augmented surgery in the surgical management of esotropia associated with hypermetropia: one-year surgical outcomes. J AAPOS 2001; 5(1): 31-34.

2 von Noorden GK, Campos EC. Binocular Vision and Ocular Motility, 6th ed. Mosby: St. Louis, 2002.

3 Havertape SA, Whitfill CR, Cruz OA. Early-onset accommodative esotropia. J Pediatr Ophthalmol Strabismus 1999; 36(2): 69-73.

4 Koc F, Ozal H, Yasar H, Firat E. Resolution in partially accomodative esotropia during occlusion treatment for amblyopia. Eye 2006; 20(3): 325-328.

5 Hiatt RL. Medical management of accommodative esotropia. J Pediatr Ophthalmol Strabismus 1983; 20(5): 199-201.

6 Miller JE. A comparison of miotics in accommodative esotropia. Am J Ophthalmol 1960; 49: 1350-1355.

7 Birch EE, Stager Sr DR, Berry P, Leffler J. Stereopsis and long-term stability of alignment in esotropia. J AAPOS 2004; 8(2): 146-150.

8 Weakley Jr DR, Birch E, Kip K. The role of anisometropia in the development of accommodative esotropia. J AAPOS 2001; 5(3): 153-157.

9 Berk AT, Kocak N, Ellidokuz H. Treatment outcomes in refractive accommodative esotropia. J AAPOS 2004; 8(4): 384-388.

10 Birch EE, Fawcett SL, Morale SE, Weakley Jr DR, Wheaton DH. Risk factors for accommodative esotropia among hypermetropic children. Invest Ophthalmol Vis Sci 2005; 46(2): 526-529.

11 Swan KC. Accommodative esotropia long range follow-up. Ophthalmology 1983; 90(10): 1141-1145.

12 Kushner BJ. Partly accommodative esotropia. Should you overcorrect and cut the plus? Arch Ophthalmol 1995; 113(12): $1530-1534$.

13 Fawcett SL, Birch EE. Risk factors for abnormal binocular vision after successful alignment of accommodative esotropia. J AAPOS 2003; 7(4): 256-262. 